\title{
Tweeting Authors: Impact on Research Publicity and Downstream Citations
}

\author{
Keith Gunaratne, M. D. ', Hourmazd Haghbayan, M.D. ${ }^{2}$, and Eric Anthony Coomes, \\ M.D. ${ }^{7}$
}

'Department of Medicine, University of Toronto, Toronto, ON, Canada; ${ }^{2}$ Division of Cardiology, London Health Sciences Centre, Western University, London, ON, Canada.

J Gen Intern Med 35(6): 1926-7

DOI: $10.1007 / \mathrm{s} 11606-019-05454-0$

(c) Society of General Internal Medicine 2019

\section{INTRODUCTION}

The increasing ubiquity of social media has revolutionized medical and scientific communication, with considerable emerging interest in the use of Twitter for research dissemination $(1,2)$. Over the past decade, the field of Pulmonary and Critical Care Medicine has innovatively applied this technology to build international research communities and promote knowledge translation (3). Previous studies have determined that highly tweeted articles are well-cited (4), and consequently some journals have begun requiring peer-reviewed journalissued tweets to accompany publications (5). While researchers have a clear incentive to promote their work, their individual potential to impact its dissemination via Twitter is unclear and the impact of direct author tweeting on citations has not been previously studied. We therefore sought to determine if articles published in Pulmonary and Critical Care Medicine journals tweeted by their authors received greater downstream tweets and citations compared with non-author-tweeted articles.

\section{METHODS}

Eighty-four Pulmonary and Critical Care Medicine journals and their 2017 Journal Impact Factor (IF) rankings were identified using the InCites Journal Citation Reports tool (Clarivate Analytics, 2018). From June 6, 2011, to January $1,2017,15,078$ articles from these 84 journals that were referenced by at least one Twitter post ("tweet") within the first year of publication were identified using Altmetric.com. Article author names were matched to Twitter users to determine whether each article was mentioned by a tweet issued by at least one author. The Web of Science database (Clarivate Analytics, 2018) was cross-referenced to identify articles'

$\overline{\text { Keith Gunaratne and Hourmazd Haghbayan are co-first authors. These }}$ authors contributed equally to this manuscript.

Received August 30, 2019

Revised August 30, 2019

Accepted September 27, 2019

Published online October 25, 2019 downstream citations. The total number of tweets at one year after publication, number of citations at one year, and total citation count (number of citations by the end of the study follow-up period on December 5, 2018) were calculated for each article. An alpha of 0.05 was pre-specified as the threshold for statistical significance; bootstrapping was used to generate $95 \%$ confidence intervals $(95 \% \mathrm{CI})$ and $p$ values for comparisons between author-tweeted and non-author-tweeted articles. Journals were stratified by IF into IF $<2$ (low-IF), IF $\geq$ 2 and $<5$ (moderate-IF), and IF $\geq 5$ (high-IF) categories. Anaconda Python 3.6 (Anaconda, Inc., Austin, TX) was used for data management and statistical analysis.

\section{RESULTS}

We identified 15,078 articles published in 84 pulmonary and critical care journals which had complete tweet and citation data (Table 1). Overall, author-tweeted articles achieved a 3.08-fold (95\% CI, 2.69-3.51; $p<0.001)$ increase in downstream tweets compared with non-author-tweeted articles (Table 1). This corresponded to a 1.41 -fold $(95 \% \mathrm{CI}, 1.25-1.58 ; p<0.001)$ increase in citations at one year and a 1.51 -fold $(95 \%$ CI, 1.32 $1.72 ; p=0.001)$ increase in total citations. Statistically significant increases in tweets at one year were observed across IF categories among author-tweeted compared with non-author-tweeted articles ( $p$ values $<0.001$ ); however, only articles in high-IF journals attained significant increases in citations (Table 1).

\section{DISCUSSION}

Our study demonstrates that articles tweeted by at least one of their authors achieved a threefold increase in tweets at one year compared with non-author-tweeted articles and that such articles went on to obtain a $50 \%$ greater number of total downstream citations. In sub-group analyses, while significant increases in tweet volumes were observed across all IF categories, this "author boost" only translated into increased downstream citations for articles in high-IF journals. The mechanism underlying these findings is unclear; one possibility is that authors on social media participate in networks of individuals with similar interests, thereby allowing greater penetrance of their tweets within such networks compared 
with tweets originating outside. Further study is required to precisely delineate the nature of this interaction.

While this is the first study, to our knowledge, to demonstrate significant association between author tweeting and frequency of article citations, this work has several limitations. The retrospective nature of our analyses on this prospectively collected, observational data precludes assertion of a direct causative link between author tweets and downstream citations. Some authors may not identify themselves on Twitter, potentially introducing information bias. It was not possible to differentiate between author-driven as opposed to journalmandated tweet issuance.

Overall, this study highlights Twitter's role in the online sharing of scientific literature, suggesting its use may represent an opportunity for researchers to significantly impact the dissemination and citation of their work. Journal editors may thus consider encouraging their authors to engage with social media to maximize the distribution of new publications and potentially promote their integration into future research articles as citations.

\section{Acknowledgments:}

The authors would like to thank Altmetric.com for providing unrestricted access to their full database free of charge for the purpose of this study.

Contributor Statement: K.G., H.H., and E.A.C designed the study. K.G. acquired and analyzed the data. K.G., H.H., and E.A.C. wrote the manuscript. All authors edited the manuscript and reviewed it critically for important intellectual content.

Corresponding Author: Eric Anthony Coomes, M.D.; Department of Medicine University of Toronto, Suite RFE 3-805, 200 Elizabeth Street, Toronto, ON M5G 2C4, Canada (e-mail: eric.coomes@mail.utoronto.ca).

\section{Compliance with Ethical Standards:}

Conflict of Interest: The authors declare that they do not have a conflict of interest.

Disclaimer: Altmetric.com was not involved in the design, conduct, or data analysis of this study, in the writing or editing of the manuscript, nor in the decision to proceed to publication.

\section{REFERENCES}

1. Moorhead SA, Hazlett DE, Harrison L, Carroll JK, Irwin A, Hoving C, et al. A New Dimension of Health Care: Systematic Review of the Uses, Benefits, and Limitations of Social Media for Health Communication. https://www. ncbi.nlm.nih.gov/pubmed/23615206

2. Antheunis ML, Tates K, Nieboer TE. Patients' and health professionals' use of social media in health care: Motives, barriers and expectations. Patient Educ Couns. 2013;92(3):426-31.

3. Barnes SS, Kaul V, Kudchadkar SR. 2018Social Media Engagement and the Critical Care Medicine Community. J Intensive Care Med. 088506661876959. https://www.ncbi.nlm.nih.gov/pubmed/29699469

4. Eysenbach G. Can tweets predict citations? Metrics of social impact based on Twitter and correlation with traditional metrics of scientific impact. J Med Internet Res. 2011;13(4).

5. Kochanek PM, Kudchadkar SR, Kissoon N. Guiding Pediatric Critical Care Medicine Toward a Bigger "Impression" in 2017 and Beyond. Pediatr Crit Care Med. 2017 May;18(5):403-4.

Publisher's Note Springer Nature remains neutral with regard to jurisdictional claims in published maps and institutional affiliations. 